\title{
LIGHTING OPTIMISATION CONTROL OF FLUO/LED SYSTEMS USING NEURAL NETWORK AND MATHEMATICAL MODEL
}

\author{
Merimé SOUFFO TAGUEU* and Benoît NDZANA \\ Laboratoire de Génie Electrique, Mécatronique et Traitement du signal, National Advanced \\ School of Engineering, University of Yaoundé I, Yaoundé, Cameroon \\ *Corresponding Author E-mail: souffotagueu@yahoo.fr
}

\begin{abstract}
This article proposes a comparison of different approaches in the lighting optimization process. Beginning with the dual Fluo/LED luminaires, a neural network model is designed to represent the relationship between dimming levels of luminaires and illumination. A similar model is built with a mathematical approach and the obtained results from all these models are compared with DIALux simulation. The proposed models, implemented in a specific room, show significant energy savings according to users preference illumination. The error generated by the models is low compared to the experimental one. The proposed approach is easy to implement in a microcontroller system and is applicable in all interior building.
\end{abstract}

Keywords: Illumination, Mathematical model, Neural network model, Dimming level, Energy savings

Cite this Article: Merimé SOUFFO TAGUEU and Benoît NDZANA, Lighting Optimisation Control of FLUO/LED Systems Using Neural Network and Mathematical Model, International Journal of Electrical Engineering \& Technology, 10(4), 2019, pp. 47-59.

http://iaeme.com/Home/issue/IJEET?Volume=10\&Issue=4

\section{INTRODUCTION}

The increase of population is in phase with energy demand. In building, lighting is energy intensive since the energy demand for lighting represents a percentage of $25-35 \%$ of the total energy consumption of a building [1]. In Africa, this percentage is larger, about $56 \%$ [2]. Such statistics invites us to revisit our consumption patterns or, to rethink the way in which buildings, schools and homes are built. Clearly, a novel mindset on our daily habits in consuming energy should be defined.

Various studies about energy savings have been performed. In [3,4], a simple analysis method is used to evaluate the potential energy savings associated with electrical lighting when it is combined with daylight use. A fuzzy controller was developed in [5] to maintain the lighting illuminance level suitable for robotic manipulation in specific environments. The 
same approach was developed in [6], where a fuzzy controller was designed to save lighting energy based on user displacements. In [7], the authors have implemented neural networks to find an optimal position of some luminaires. In [8], lighting control has been modeled as a linear programming problem to accomplish energy efficiency and satisfy occupants' lighting preferences. In [9], a feed-forward neural network model was proposed to describe the relationship between the dimming level of LED luminaires and the illumination on a table without using simulation software. The actuation command to the luminaires was generated by a feed forward neural network control strategy. Some researchers in [10], use simulation software to carry out the lighting configuration in the test room to establish the relationship between lights and table illuminance. However, to the best of our knowledge, there is no work in the literature taking into account the mathematical model relationship between illumination and dimming level of luminaire compared to other methods such as software simulation and neural network model.

In this article, a comparison of different approach in lighting optimization process is proposed. This study considers two differents models built mathematically and with a trained neural network, in other to show a relevant reduction in percentage of dimming level of the luminaires. The article is organized as follows: in section 2, the test room in initial form with fluorescent luminaire and final form with LED luminaire is presented, followed by a mathematical model and neural network model. Also, implementations of the proposed optimization method are provided. Section 3 presents a case study where the methods explained in the previous section are implemented to have the desired levels of illumination. The lighting optimization solution is shown in section 4 while conclusions are drawn in section 5 .

\section{METHODOLOGY}

\subsection{Fluo/LED lighting test room}

The test room is an office room with dimensions of $5.5 \times 4.5 \times 2.8 \mathrm{~m}$. The fluorescent luminaire installed have the characteristics shows in Table 1. The Fluo test room and the visual arrangement of the luminaires insides the office room are shown respectively in Figure 1a and Figure $1 \mathrm{~b}$.

Table 1 Fluorescent luminaire TBS 165 G 4xTL5-14W HFS C6 specifications.

\begin{tabular}{|l|l|}
\hline Visual form & \\
Light Output Radio & 0.70 \\
Total luminaire power & $61 \mathrm{~W}$ \\
Total lamp flux & $48001 \mathrm{~m}$ \\
Luminous flux & $33601 \mathrm{~m}$ \\
Technology & FLUO \\
Manufacturer code & TBS 165 \\
\hline
\end{tabular}




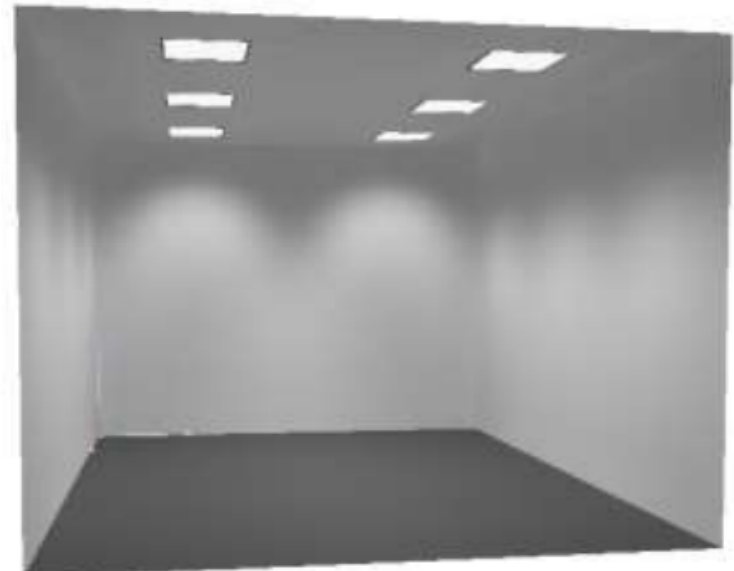

(a)

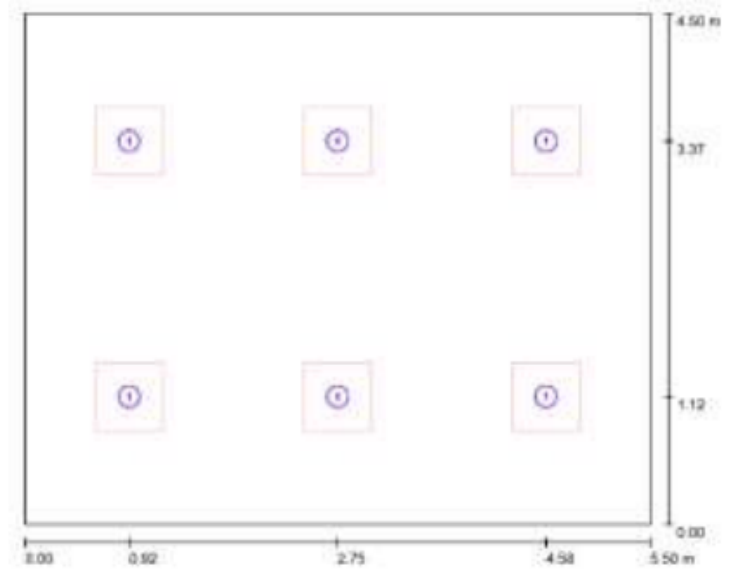

(b)

Figure 1: (a) Fluo test room. (b) Visual form of the office and arrangement of the luminaires insides the room.

The objective is to design the relationship between the dimming level of the luminaire and the illuminance. In the installed room, we note that the luminaire is fluorescent technologies and to have a better result we will choose a LED luminaire with are more interesting because of their low power consumption and the fact that there are easily dimmable.

The LED luminaire RC480 W60L60 PCV 1x LED35S/840 AC-MLO of constructor PHILIPS are chosen for experimentation. Their specifications are shown in Table 2, while Figure 2 shows its image on the market. These luminaires are DALI controlled. The Digital Addressable Lighting Interface (DALI) is a standardized protocol (IEC 62386) for digital communication between lighting fixtures being addressed individually [11]. All the LED luminaire used are dimmable and can be controlled by a central controlling computer. The dimming level of a luminaire is proportional to its power consumption. The implantation principle of the luminaire in an office room is the same as in Figure $1 \mathrm{~b}$.

Table 2 LED Luminaire specifications.

\begin{tabular}{|l|l|}
\hline Light Output Radio & 1.00 \\
Total luminaire power & $30 \mathrm{~W}$ \\
Total luminaire flux & $35001 \mathrm{~m}$ \\
Technology & LED \\
Dimmable & DALI \\
Manufacturer code & RC 480 \\
\hline
\end{tabular}

Figure 2 LED luminaire RC480 W60L60 PCV 1x LED35S/840 AC-MLO. 


\subsection{Model formulation}

\subsubsection{Mathematical problem formulation}

There are two mains characteristics in an interior lighting namely illuminance and uniformity of the lighting. Both characteristics are normalized using EN 12464 standard [12] henceforth, turns all optimization efforts to lie within the specifications of the corresponding norm. It is worth to recall that our goal is to minimize energy consumption by reducing the dimming level of the luminaires in an interior building. Based on the superposition theory in physics, the illuminance at a specific point of the work plane level $\left(E_{i}\right)$, is equal to the contribution of each luminaire on that measurement point $\left(\omega_{\mathrm{ij}}\right)$ and the corresponding dimming level of that luminaire $\left(\alpha_{j}\right)[13,14,15,16]$. Representing the illuminance on each measurement point, the dimming of the luminaires also as a vector $(\alpha)$ and the contribution of each luminaire to each measurement point as a matrix $(\omega)$, the model can be formulated as shown below :

$$
E=\left[\begin{array}{c}
E_{1} \\
E_{2} \\
\vdots \\
E_{M}
\end{array}\right]=\left[\begin{array}{ccc}
\omega_{11} & \cdots & \omega_{1 N} \\
\vdots & \ddots & \vdots \\
\omega_{M 1} & \cdots & \omega_{M N}
\end{array}\right]\left[\begin{array}{c}
\alpha_{1} \\
\alpha_{2} \\
\vdots \\
\alpha_{N}
\end{array}\right]=\omega \times \alpha
$$

We can also rewrite equation 1 as follow :

$$
E_{i}=\sum_{j=1}^{N} \alpha_{j} \omega_{i j}
$$

Where $\mathrm{N}$ is the number of luminaire and $\mathrm{i}$ depend on the number of measurements points $\mathrm{M}$.

The mean illuminance, according to equation (2) is :

$$
E_{m}=\frac{\sum_{i=1}^{M} E_{i}}{M}=\frac{\sum_{i=1}^{M} \sum_{j=1}^{N} \alpha_{j} \omega_{i j}}{M}
$$

To find the contribution of each luminaire, a simulation tool is used, but another method was to used a luxmeter at different choice point [13]. The luminaire needs to be already installed when the second method is used. To have the dimming level, we can modify equation 1 as :

$$
\alpha=\left[\begin{array}{c}
\alpha_{1} \\
\alpha_{2} \\
\vdots \\
\alpha_{n}
\end{array}\right]=\left[\begin{array}{ccc}
\omega_{11} & \cdots & \omega_{1 m} \\
\vdots & \ddots & \vdots \\
\omega_{n 1} & \cdots & \omega_{n m}
\end{array}\right]^{-1}\left[\begin{array}{c}
E_{1} \\
E_{2} \\
\vdots \\
E_{n}
\end{array}\right]=\omega^{-1} \times E
$$

Computing the pseudo matrix $\omega^{-1}$, we can rewrite equation 4 as :

$$
\alpha=\left[\begin{array}{c}
\alpha_{1} \\
\alpha_{2} \\
\vdots \\
\alpha_{n}
\end{array}\right]=\left[\begin{array}{ccc}
c_{11} & \cdots & c_{1 N} \\
\vdots & \ddots & \vdots \\
c_{1 M} & \cdots & c_{N M}
\end{array}\right]\left[\begin{array}{c}
E_{1} \\
E_{2} \\
\vdots \\
E_{N}
\end{array}\right]=c \times E
$$

Where $\mathrm{c}=\omega^{-1}$ and $0 \leq \alpha \leq 1$

Equation 5 represents the mathematical representation of the dimming level of the luminaire in function of illuminance $\mathrm{E}$.

The uniformity $\mathbf{g}_{\mathbf{0}}$ is defined as :

$g_{0}=\frac{E_{\min }}{E_{m}}$

Where $\mathrm{E}_{\min }$ is the minimum value of illumination.

\subsubsection{Neural Network model}

Artificial neural networks (ANN) are computing systems that are inspired by, the biological neural networks that constitute animal brains. Theses types of systems "learn" to perform 
tasks by considering examples, generally without being programmed with any task-specific rules. Figure 3 shows the principle/specification of our model.

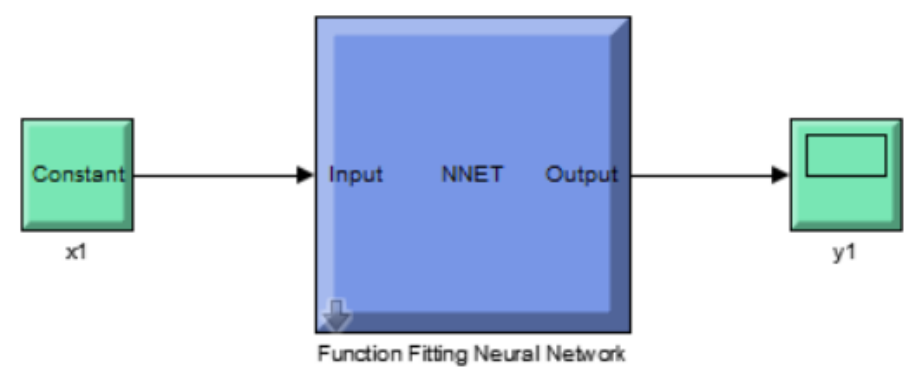

Figure 3 ANN model principle.

There are many algorithms (gradient descent, quasi-Newton method, LevenbergMarquardt, just to name few) used to design a neural network system according to the dimension of train data. In our model, we have many neural networks to train with just a few thousands of instances and a few hundreds of parameters, the best choice might be the Levenberg-Marquardt algorithm.

The Levenberg-Marquardt algorithm is a method tailored for functions of the type sum-ofsquared-error. That makes it to be very fast when training neural networks measured on that kind of errors. The Levenberg-Marquardt algorithm, also known as the damped least-squares method, has been designed to work specifically with loss functions which take the form of a sum of squared errors. It works without computing the exact Hessian matrix. Instead, it works with the gradient vector and the Jacobian matrix. [17]

Figure 4 represents a state diagram for the training process of an artificial neural network with the Levenberg-Marquardt algorithm. The first step is to calculate the loss, the gradient and the Hessian approximation. Then the damping parameter is adjusted so as to reduce the loss at each iteration.

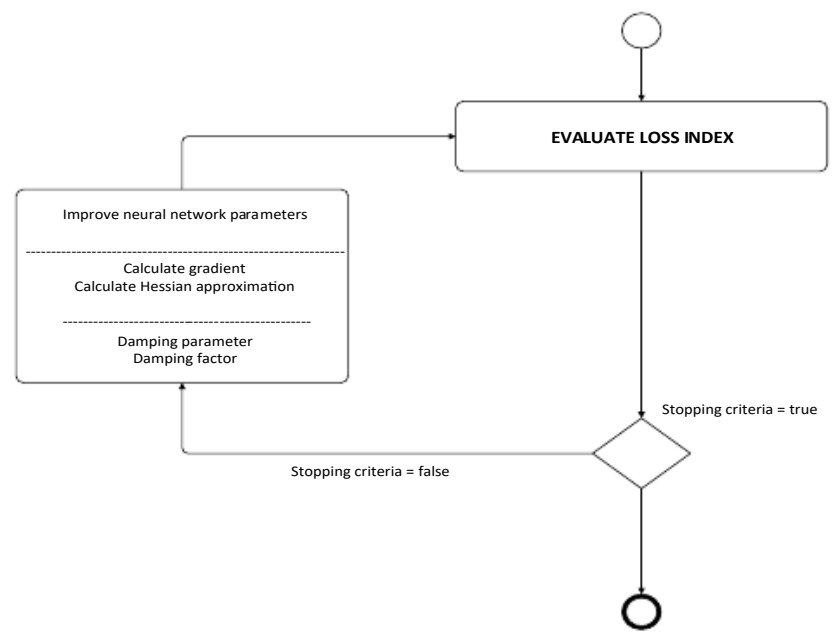

Figure 4 State diagram for the training process of a neural network with the LevenbergMarquardt algorithm. 


\section{IMPLEMENTATION OF THE METHODOLOGY}

\subsection{Office simulation}

DIALux [18] which is a simulation tool that is used for electric lighting design practice and energy research is used for lighting simulation and the illumination and uniformity are fixed by the norm 12464 [12]. In these case, the recommend plage of variation of illumination is around $E_{m}=300 l x$ and $E_{m}=500 l x$ and uniformity will be up to $g_{0}=0.6$. The Light loss factor is choice 0.8 and the Height of working plane around $0.800 \mathrm{~m}$. Figure 5 shows the illumination isolines without specific optimization and the result obtain give $E_{m}=542 l x$ and $g_{0}=0.669$.

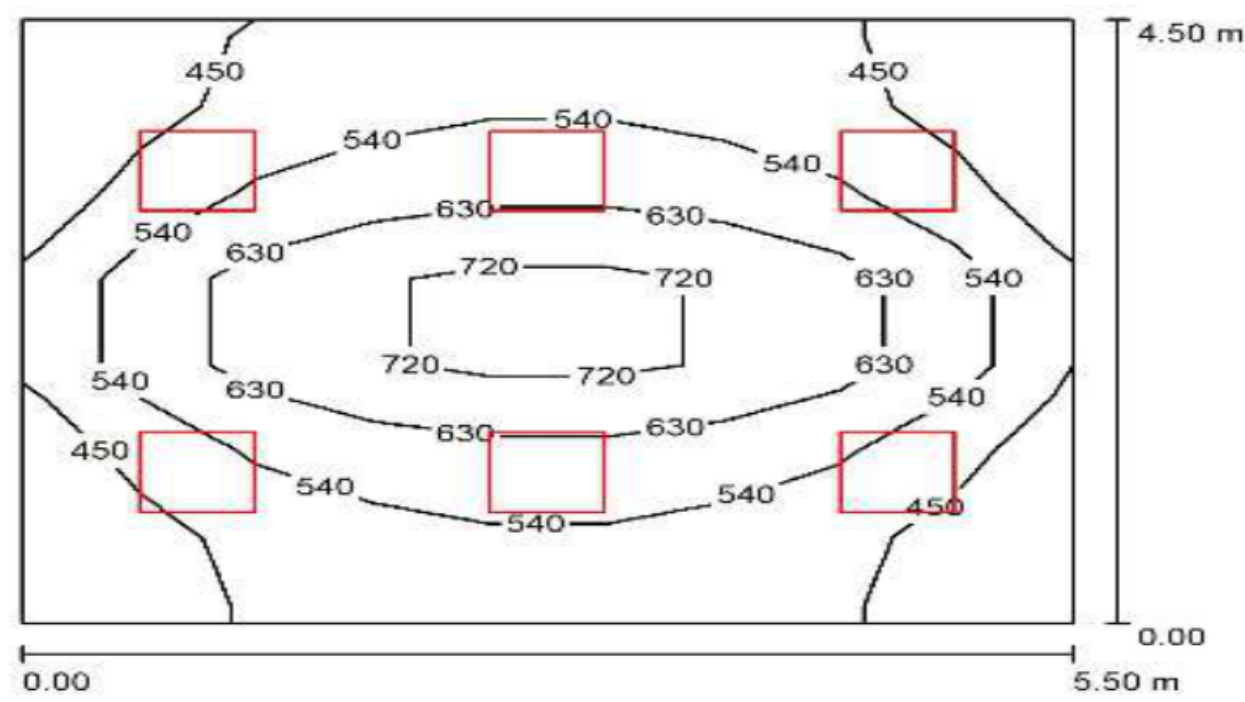

Figure 5 Illumination isoline of the office.

\subsection{Optimization procedure using mathematical model}

The first step is to define the quantity of point of the table of illuminance and then find the corresponding illuminance using a luxmeter integrated into a DIALux software. Table 4 gives the parameter used in our modelization. The mathematical formulation of the proposed model which is adapted to the arrangement of the above mentioned office room is presented in equation 6.

$$
\alpha=\left[\begin{array}{c}
\alpha_{1} \\
\alpha_{2} \\
\alpha_{3} \\
\alpha_{4} \\
\alpha_{5} \\
\alpha_{6}
\end{array}\right]=c \times E=\left[\begin{array}{ccc}
c_{11} & \cdots & c_{63} 1 \\
\vdots & \ddots & \vdots \\
c_{16} & \cdots & c_{636}
\end{array}\right]\left[\begin{array}{c}
E_{1} \\
E_{2} \\
\vdots \\
E_{63}
\end{array}\right]
$$

With $0 \leq \alpha \leq 1$

Table 4 Model parameter

Number of luminaires (M) 6

Dimming level number 6

Number of measurement points in $\mathrm{x}$ axis $\quad 9$

Number of measurement points in y axis 6

Total Number of measurement points $(\mathrm{N}) \quad 63$ 
The office room is divided into 63 zones representing by a measured point « $\mathrm{E}_{\mathrm{i}}$ ». The repartition of the measured illuminance point is shown in Figure 6.

\begin{tabular}{|c|c|c|c|c|c|c|c|c|}
\hline E1 & E2 & E3 & E4 & E5 & E6 & E7 & E8 & E9 \\
\hline E10 & E11 & E12 & E13 & E14 & E15 & E16 & E17 & E18 \\
\hline E19 & E20 & E21 & E22 & E23 & E24 & E25 & E26 & E27 \\
\hline E28 & E29 & E30 & E31 & E32 & E33 & E34 & E35 & E36 \\
\hline E37 & E38 & E39 & E40 & E41 & $E 42$ & E43 & E44 & E45 \\
\hline E46 & E47 & E48 & E49 & E50 & E51 & E52 & E53 & E54 \\
\hline E55 & E56 & E57 & E58 & E59 & E60 & E61 & E62 & E63 \\
\hline
\end{tabular}

Figure 6 Table of illuminance

\subsection{Optimization procedure using neural network model}

A Two-layer feed-forward neural network, developed in Matlab 2014a [19], is used for mapping between a data set of numeric inputs, dimming levels of 6 luminaires and a set of numeric targets, the corresponding illuminance of 63 points given the dimming setting. Thus, the network is with 63 inputs and 6 outputs, shown in Figure 7.

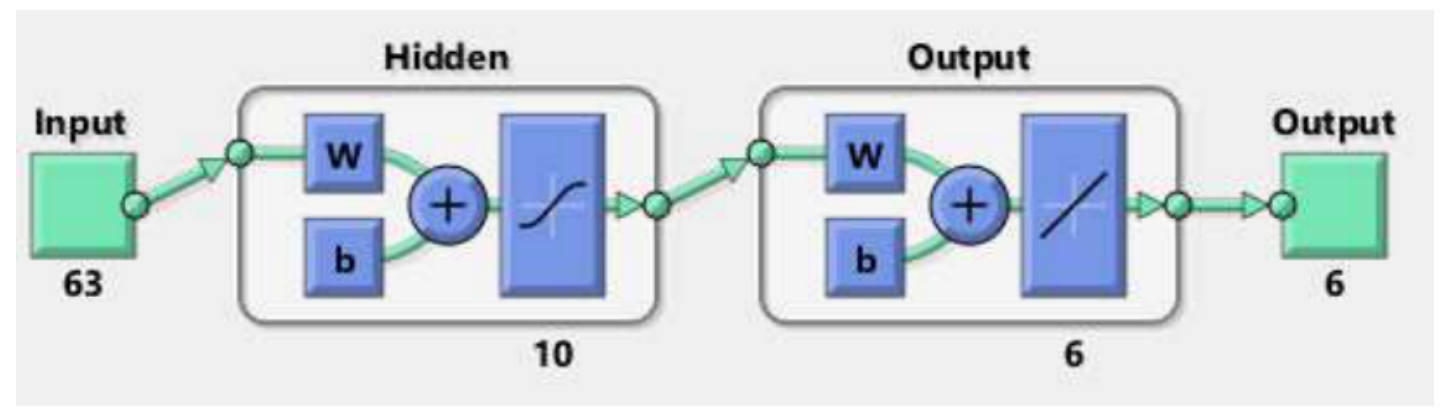

Figure 7 The two-layer feed-forward network.

After defining the input and output of our neural network model, the design process can be done. The first step is to collect the data. In this phase, we use about a hundred random set of input data. These samples points are obtained with DIALux simulation. The dimming level of the luminaire is taken between $50 \%$ and $100 \%$ and as parameter of the hidden layer, we use a hyperbolic tangent sigmoid transfer function.

The second step is the division of data. With these settings, the input vectors and target vectors will be randomly divided into three sets as follows:

- $70 \%$ will be used for training.

- $15 \%$ will be used to validate that the network is generalizing and to stop training before overfitting.

- The last $15 \%$ will be used as a completely independent test of network generalization.

The third step is the train of the network. The training process requires a set of examples of proper network behavior - network inputs $\mathrm{c}$ and target outputs t. The process of training a neural network involves tuning the values of the weights $W$ and biases $b$ of the network to optimize network performance. The performance function for feedforward networks is mean 
square error (MSE) - the average squared error between the network outputs $\alpha$ and the target outputs t. The curve of the calculated MSE during the training process is shown in Figure 8. The MSE is defined in equation 7.

$$
M S E=\frac{1}{N} \sum_{1}^{N}\left(t_{i}-\alpha_{i}\right)^{2}
$$

The last step is to create and initialize the network. A two-layer feed-forward neural network with $\mathrm{M}$ neurons in the hidden layer is created. The weights and biases of the network are also initialized. The regression of the network is shown in Figure 9.

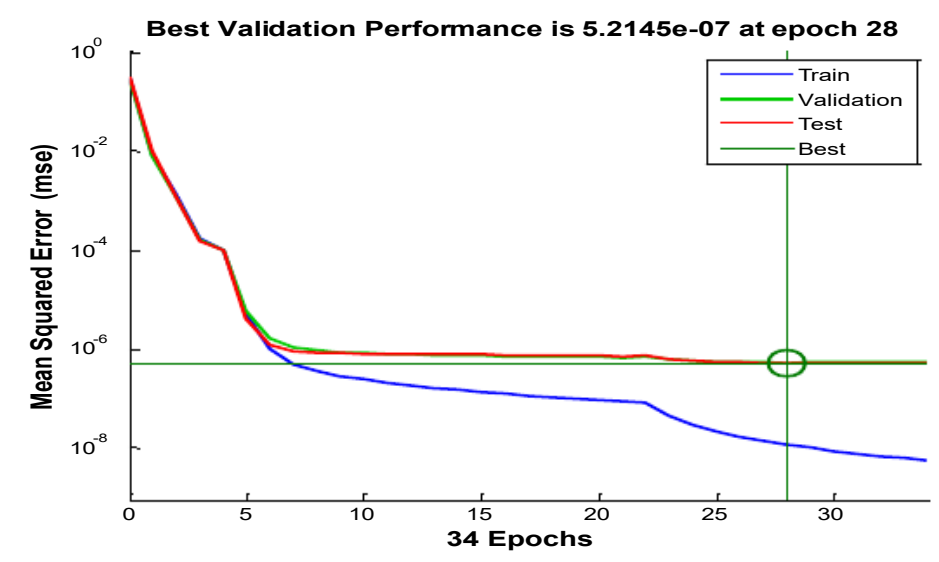

Figure 8 The best validation performance of the trained network
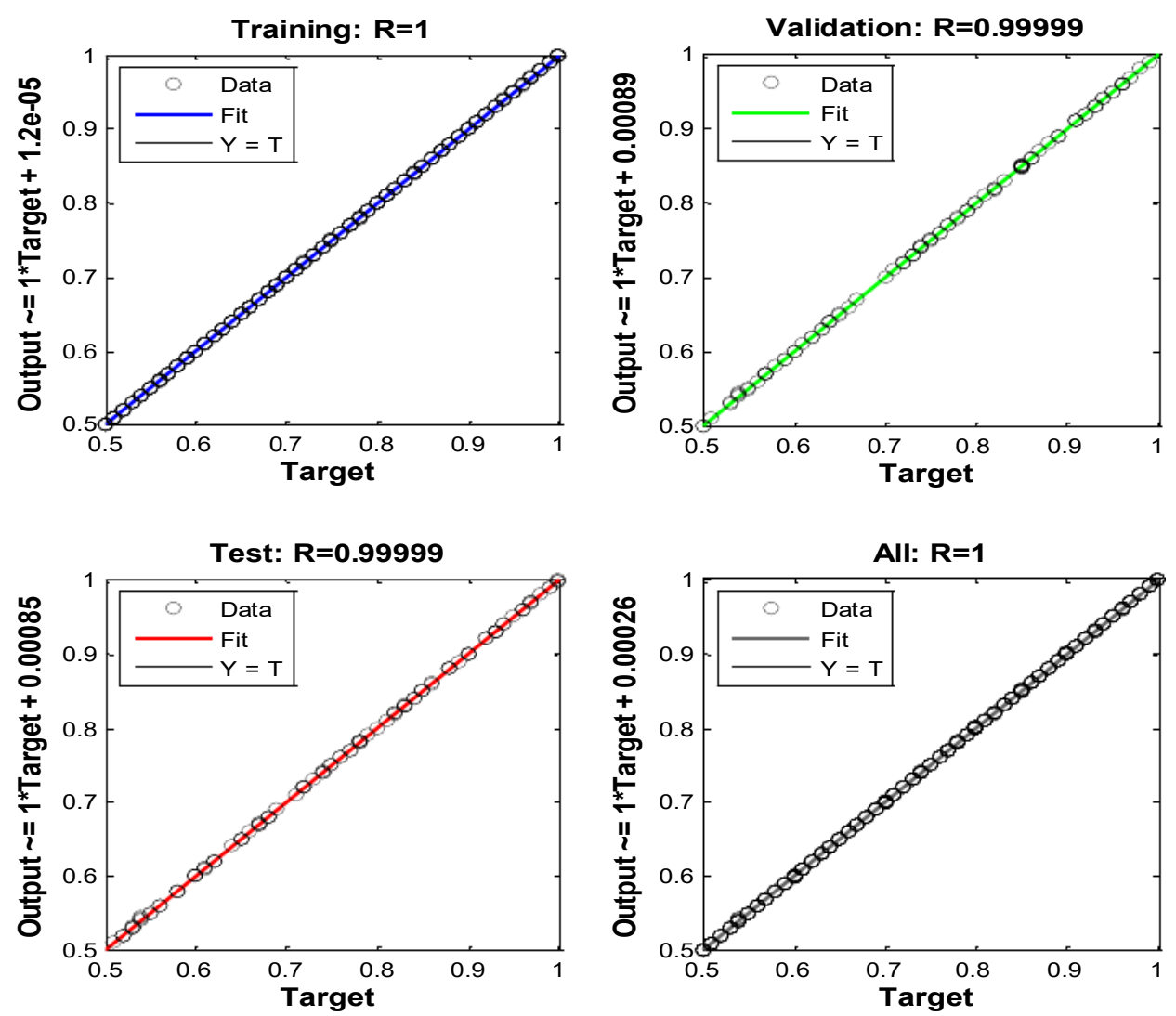

Figure 9 The regression result of the trained network. 


\section{RESULTS AND DISCUSSIONS}

The simulation of the lighting levels is carried out in DIALux. The different models designed allow us to conduct our experiment. Since the initial value of the overall illumination of the part is $\mathrm{E}_{\mathrm{m}}=5421 \mathrm{x}$, users lighting preferences are set to have the corresponding coefficients of variation for each of the implemented models. These preferences are chosen in the range from 3001x to 5001x, accepted by the standard EN12464 and tested on the models developed in the implementation part of the methodology. The isolines curves obtained for each case are shown in Figure 10. For each set illumination value, the illumination table is obtained by subtracting for each level of illumination Eij the value to be removed from the initial illumination for having the desired illumination. Thus, for the reference 5001x for example, we must subtract from each of the 63 measuring points the excess amount of illumination ie about 421x. The same procedure is applied for the different other references. The introduction of these illuminance values in our models gives the coefficients of variation presented in Table 5 . We can already see that the dimming level values obtained by each model are close. The actual illumination values given by these coefficients of variation calculated by our models are then verified by DIALux simulation. The values obtained by simulation are recorded in Table 6. By calculating the error between the different models, it is found that it is very small, a few lux more or less than the reference value. We can therefore conclude that the values that the users would set using the models designed will be very close to reality and therefore the models can be used in practice. Moreover, we see that the results of the mathematical model are closer to those obtained by simulation than those of the neural network model. This can be explained by the not very large number of points used to train the network.

The ultimate objective sought is the reduction of energy consumption, Table 7 presents for the desired illumination references and the range of variation of this economy in power consumed which ranges from about $8 \%$ to $45 \%$. It can therefore be concluded that according to users needs, energy savings can be very high.

These two models are interesting because they can be easily implemented in a microcontroller and therefore with a graphical interface on a computer. The user following these needs can set levels of illumination as he wishes. Given that these levels of illumination will be lower than the level for which the illumination is maximum, a reduction in the consumption of electrical energy will necessarily be achieved. The only constraint of the system is the knowledge beforehand of the different lighting points when all the levels of illumination are set to $100 \%$ for the mathematical model and to have a number of test points for the training of the system by neural network.

Table 5 User preference illumination and corresponding dimming level of luminaires

\begin{tabular}{lccccccccccc}
\hline & \multicolumn{4}{c}{ MATHEMATICAL MODEL } & \multicolumn{6}{c}{ NEURAL NETWORK MODEL } \\
\hline Users preference & 300 & 350 & 400 & 425 & 500 & 300 & 350 & 400 & 425 & 500 \\
illuminance (lux) & & & & & & & & & & \\
$\alpha_{1}$ & 0.44 & 0.55 & 0.67 & 0.72 & 0.89 & 0.34 & 0.42 & 0.52 & 0.59 & 0.86 \\
$\alpha_{2}$ & 0.44 & 0.56 & 0.67 & 0.72 & 0.89 & 0.45 & 0.56 & 0.68 & 0.73 & 0.90 \\
$\alpha_{3}$ & 0.78 & 0.83 & 0.88 & 0.90 & 0.98 & 0.69 & 0.77 & 0.85 & 0.89 & 0.98 \\
$\alpha_{4}$ & 0.78 & 0.83 & 0.88 & 0.90 & 0.98 & 0.96 & 1 & 1 & 1 & 1 \\
$\alpha_{5}$ & 0.44 & 0.55 & 0.67 & 0.72 & 0.89 & 0.45 & 0.52 & 0.60 & 0.87 & 0.87 \\
$\alpha_{6}$ & 0.45 & 0.56 & 0.67 & 0.72 & 0.89 & 0.48 & 0.60 & 0.72 & 0.91 & 0.91 \\
\hline
\end{tabular}


Merimé SOUFFO TAGUEU and Benoît NDZANA

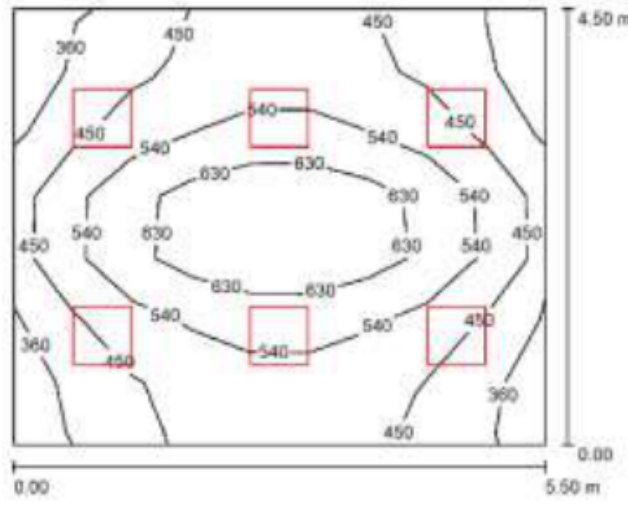

(a)

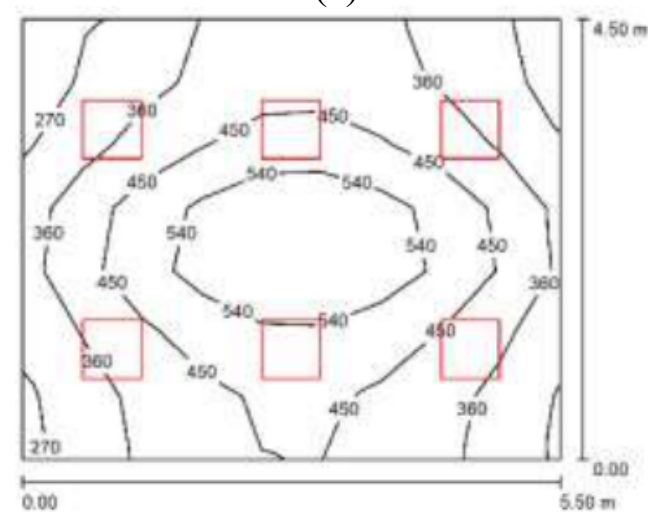

(c)

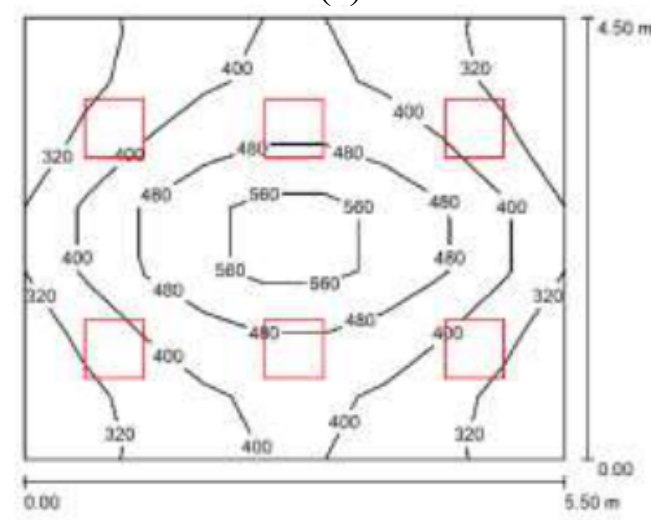

(e)

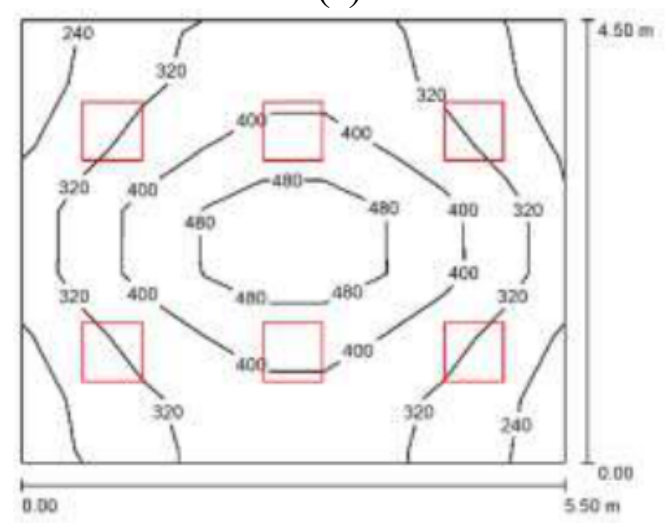

(g)

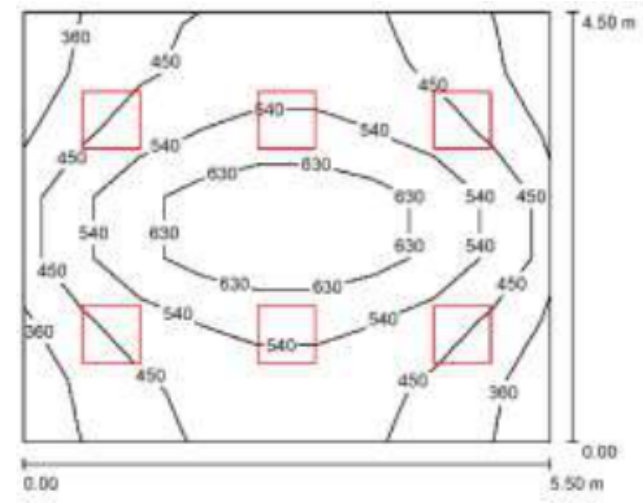

(b)

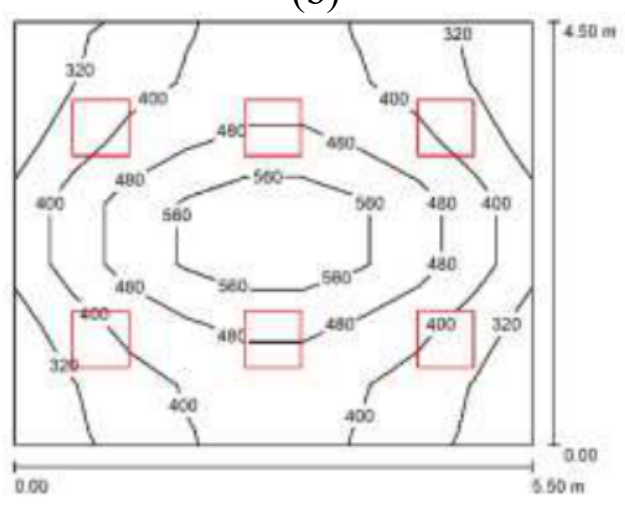

(d)

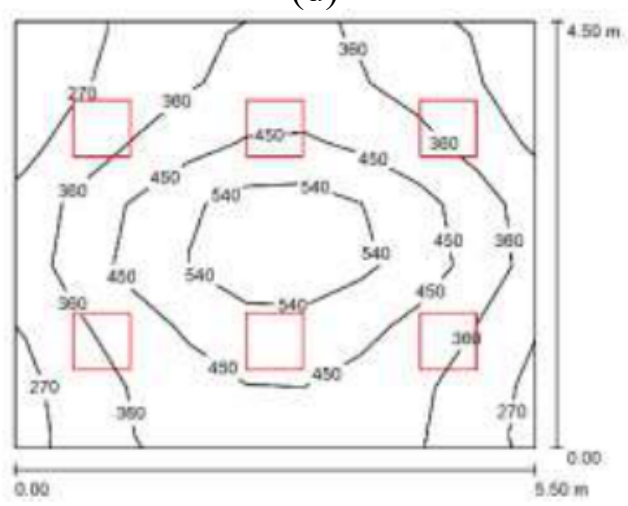

(f)

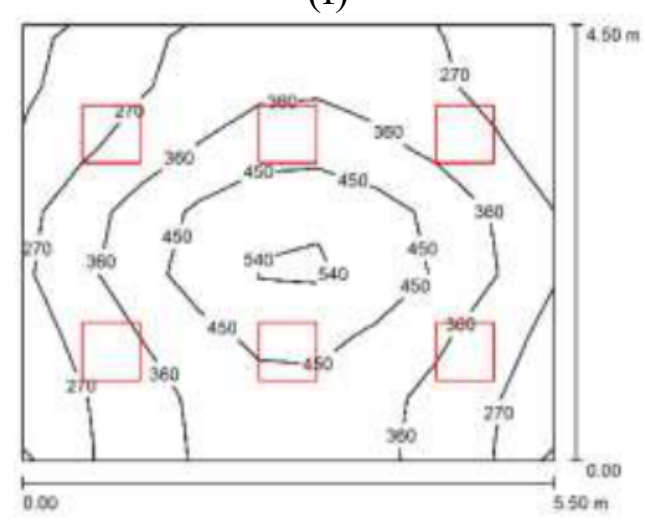

(h) 


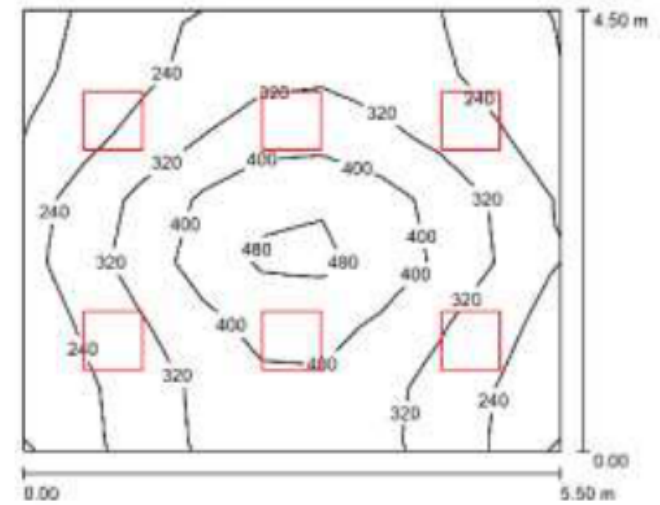

(i)

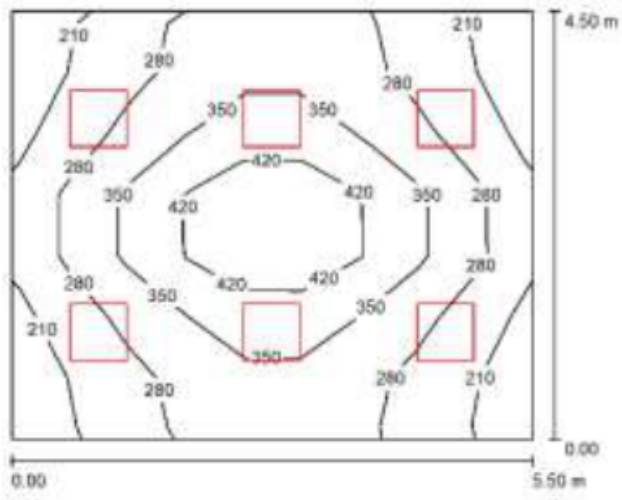

(j)

Figure 10 Isoline under user preference illuminance (a) target of 5001x with Neural Network model (b) target of 5001x with Mathematical model (c) target of 4251x with Neural Network model model (d) target of 4251x with Mathematical model (e) target of 4001x with Neural Network model model (f) target of 4001x with Mathematical model (g) target of 3501x with Neural Network model model (h) target of 3501x with Mathematical model (i) target of 3001x with Neural Network model model (j) target of 3001x with Mathematical model.

Table 6 Parameter value after optimization and corresponding light simulation

\begin{tabular}{lcccccccccc}
\hline & \multicolumn{1}{c}{ MATHEMATICAL MODEL } & \multicolumn{7}{c}{ NEURAL NETWORK MODEL } \\
\hline $\begin{array}{l}\text { Users preference of } \\
\text { illuminance (lux) }\end{array}$ & 300 & 350 & 400 & 425 & 500 & 300 & 350 & 400 & 425 & 500 \\
$\begin{array}{l}\text { Experiments values } \\
\text { of illuminance (lux) }\end{array}$ & 306 & 355 & 404 & 426 & 500 & 311 & 356 & 400 & 424 & 500 \\
Gap & 6 & 5 & 4 & 1 & 0 & 11 & 6 & 0 & -1 & 0 \\
\hline
\end{tabular}

Table 7 Optimal lighting power consumption

\begin{tabular}{cc}
\hline $\begin{array}{c}\text { Users' preference } \\
\text { (lux) }\end{array}$ & $\begin{array}{c}\text { Approximate Percentage of } \\
\text { energy saved }\end{array}$ \\
\hline $\mathbf{3 0 0}$ & $45 \%$ \\
$\mathbf{3 5 0}$ & $36 \%$ \\
$\mathbf{4 0 0}$ & $26 \%$ \\
$\mathbf{4 2 5}$ & $22 \%$ \\
$\mathbf{5 0 0}$ & $8 \%$ \\
\hline
\end{tabular}

\section{CONCLUSION}

Starting from the problem of reducing the consumption of lighting in buildings, this article highlights a comparison of different approaches in lighting optimization process. Beginning with the dual Fluo/LED luminaires, a neural network model is designed to represent the complex relationship between the coefficient of variation of luminaires and illumination. A similar model is built with a mathematical approach and the result given by all these models are compared with DIALux simulation with satisfactory results. The evaluation of error shows that the mathematical model is better than the neural network model but the two allow us to optimize lighting consumption in a specific building. The proposed model, implemented in a specific room, show significant energy savings according to user preference illumination. The gap generated by the models is low compared to the experimental one. The proposed 
approach is easy to implement in a microcontroller system and is applicable in all interior building.

\section{ACKNOWLEDGMENTS}

The first author would like to thank Dr. Theophile Fozin for many informative discussions.

\section{REFERENCES}

[1] M.-C. Dubois, ${ }^{\circ}$ A. Blomsterberg, Energy saving potential and strategies for electric lighting in future north european, low energy office buildings: A literature review, Energy and buildings 43 (10) (2011) 2572-2582.

[2] V.-Kitio, Intégration des mesures d'éfficacité énergétique et de conservation des ressources dans les normes de construction au cameroun, http://arpedac.org/wpcontent/uploads/2017/04/Projet-ONUHabitat-MiHDU-vincent-Kitio.pdf. (Accessed on april 2019).

[3] M. Krarti, P. M. Erickson, T. C. Hillman, A simplified method to estimate energy savings of artificial lighting use from daylighting, Building and Environment 40 (6) (2005) 747-754.

[4] P. Ihm, A. Nemri, M. Krarti, Estimation of lighting energy savings from daylighting, Building and Environment 44 (3) (2009) 509-514.

[5] S.Y. Chen, J.W. Zhang, Intelligent Lighting Control for Vision-Based Robotic Manipulation", IEEE Transactions on Industrial Electronics 59 (2012)3254-3263

[6] J. Liu, W. Zhang, X. Chu, Y. Liu, Fuzzy logic controller for energy 422 savings in a smart led lighting system considering lighting comfort and 423 daylight, Energy and Buildings 127 (2016) 95-104.

[7] Z. Wang, Y. K. Tan, Illumination control of led systems based on neural network model and energy optimization algorithm, Energy and Buildings 62 (2013) 514521.

[8] Y.J. Wen, A.M. Agogino, Personalized dynamic design of networked lighting for energy-efficiency in open-plan offices, Energy Build. 43 (2011) 1919-1924

[9] D. Tran, Y. K. Tan, Sensorless illumination control of a networked led-lighting system using feedforward neural network, IEEE Transactions on Industrial Electronics 61 (4) (2013) 2113-2121.

[10] A.D. Galasiu, M.R. Atif, Applicability of daylighting computer modeling in real case studies: comparison between measured and simulated daylight availability and lighting consumption, Building and Environment 37 (2002) 363-377.

[11] Dali - digital addressable lighting interface, http://www.dali-ag.org.

[12] Light and lighting of work places ? part 1: Indoor work places, european Committee for Standardization (June 2011). 
[13] E.-N. D. Madias, P. A. Kontaxis, F. V. Topalis, Application of multi-objective genetic algorithms to interior lighting optimization, Energy and Buildings 125 (2016) 66-74.

[14] Y.-J. Wen, A. Agogino, Control of wireless-networked lighting in open-plan offices, Lighting Research \& Technology 43 (2) (2011) 235-248.

[15] Y.-J. Wen, A. M. Agogino, Personalized dynamic design of networked lighting for energy-efficiency in open-plan offices, Energy and Buildings 43 (8) (2011) 19191924.

[16] M. Fischer, K. Wu, P. Agathoklis, Intelligent illumination model-based lighting control, in: 2012 32nd International Conference on Distributed Computing Systems Workshops, IEEE, 2012, pp. 245-249.

[17] Neural Designer, URL

https://www.neuraldesigner.com/blog/5_algorithms_to_train_a_neural_network.(a ccessed July 2019)

[18] Dialux documentation, https://www.dial.de/en/dialux/.

[19] Matlab 2014 documentation, https://fr.mathworks.com/products/globaloptimization.html. 